\title{
La caracterización del saber especializado en los diccionarios generales bilingües desde la perspectiva del traductor
}

\section{(The characterization of specialized knowledge in general bilingual dictionaries from Translator's perspective)}

María Teresa Ortego Antón/Purificación Fernández Nistal tortego@lesp.uva.es/purifer@itbyte.uva.es Universidad de Valladolid

Fecha de recepción: 22 de septiembre de 2014

Fecha de aceptación: 1 de noviembre de 2014

Resumen: En las últimas décadas se ha producido una revolución en el panorama del saber especializado con la aparición de nuevos conceptos y, por ende, de infinidad de denominaciones, muchas de las cuales han pasado a formar parte del acervo lingüístico de los usuarios de una lengua con un nivel cultural medio. Dicho saber especializado suele producirse en lengua inglesa, si bien surge la necesidad de transvasarlo a otras lenguas como el español. Para solventar las dificultades que aparecen durante la traducción los usuarios suelen acudir a los diccionarios generales bilingües, a pesar de que ésta no es la herramienta más aconsejable para tomar decisiones sobre el léxico especializado. En consecuencia, en el presente trabajo pretendemos obtener una caracterización de las unidades que conforman el saber experto en dos de los diccionarios generales bilingües de mayor prestigio y difusión en el mercado editorial. De los resultados que se desprendan de nuestro estudio esperamos obtener datos relevantes que incidan en una implementación de la información especializada recogida en las obras de referencia así como en los procedimientos de consulta empleados por parte de los potenciales usuarios, entre los que destacan los traductores y, especialmente, los traductores en formación.

Palabras clave: Saber especializado, Unidades de significación especializada, Diccionarios generales bilingües, Traducción.

Abstract: In recent decades, there has been a revolution in expert knowledge due to the emergence of new concepts and, consequently, new terms. Thus, general vocabulary has been gradually enlarged with terms traditionally limited to specialised discourse. In addition, expert knowledge is 
usually produced in the English language. However, in the globalised world, the need to transfer terms to other languages such as Spanish has emerged. General bilingual dictionaries are increasingly used to solve the difficulties that arise for users in the transference of specialised knowledge into other languages; nevertheless, this tool is not the most suitable when making decisions about the equivalents of specialised terms in other language. In this paper, we try to get a characterization of specialized vocabulary gathered in two of the most prestigious general bilingual dictionaries in English and Spanish. From our findings, we hope to obtain relevant data that emphasize the need to improve the standard of information gathered in these reference works and implement the searching procedures adopted by general bilingual dictionary users and, specially, by translators and translation trainees.

Key words: Specialised knowledge, Terms, General bilingual dictionaries, Translation.

\section{LA RELEVANCIA DEL LÉXICO ESPECIALIZADO EN LA SOCIEDAD ACTUAL}

En las últimas décadas se ha producido una explosión del conocimiento científico y tecnológico que ha generado un crecimiento exponencial de los conceptos especializados $y$, en consecuencia, un aumento de nuevas denominaciones, o de modificaciones de significado de las ya existentes, lo que ha obligado a los compiladores de los diccionarios generales a ampliar las nomenclaturas en las nuevas ediciones de las obras.

Además, dicho conocimiento ha pasado de ser patrimonio exclusivo de los expertos a interesar también a los usuarios de una lengua con un nivel cultural medio, fruto de la democratización que ha experimentado la educación y del papel de los medios de comunicación en la difusión de la ciencia y de la tecnología.

Por otra parte, este hecho viene acompañado de la necesidad de comunicar el saber especializado entre usuarios de diferentes lenguas y culturas, debido al carácter globalizado de nuestra sociedad. Dado que el conocimiento experto habitualmente se produce 0 se difunde internacionalmente utilizando el inglés, la lingua franca por excelencia, tanto traductores como expertos se enfrentan con la barrera de la transferencia de las unidades de significado especializado (USE). Para solventar esta dificultad, en la mayoría de las ocasiones suelen acudir a los diccionarios generales bilingües. A pesar de no ser la estrategia más recomendable, son múltiples los estudios que constatan lo generalizado de la realidad de esta conducta (Meyer, 1988; Atkins y Varantola, 1998a, 1998b; Mackintosh, 1998: 123-149; Durán Muñoz, 2010; Bowker, 2012). Sin embargo, tal y 
como prueban los mencionados estudios, la información recogida en estas obras puede inducir a errores cuando los usuarios desconocen los conceptos especializados.

En el panorama de la lexicografía bilingüe tenemos constancia de trabajos de referencia que estudian los distintos elementos que caracterizan a las USE en los pares de lenguas de inglés-francés (Jessen, 1996; Thoiron, 1998; Boulanger, 2001; Roberts, 2004; Roberts y Josselin, 2005; Josselin, 2005), de francés-español (Sierra Soriano, 2001) o de italianoespañol (Rodríguez Reina, 2002).

Sin embargo, el campo de la lexicografía general bilingüe de las lenguas inglesa y española está necesitado de estudios que profundicen en el tratamiento y la caracterización del saber especializado recogido en este tipo de obras (Ortego Antón, 2012: 66).

Basándonos en nuestra experiencia de formadoras de traductores, así como de traductoras y, por tanto, usuarias de los diccionarios generales bilingües, consideramos que el estudio de la caracterización del saber especializado incluido en estas obras puede ofrecernos una aproximación a la configuración y representación de dicho saber en los diccionarios generales bilingües. De hecho, consideramos que ésta es una parcela necesitada de estudio que puede ofrecer datos relevantes que incidan en una implementación de la información especializada recogida en las obras de referencia así como en los procedimientos de consulta empleados por parte de los potenciales usuarios, entre los que destacan los traductores y, especialmente, los traductores en formación.

2. LA DELIMITACIÓN DEL CAMPO DE APLICACIÓN: LOS DICCIONARIOS GENERALES BILINGÜES DE INGLÉS Y ESPAÑOL

Dada la enorme proliferación en el mercado editorial en los últimos años de diccionarios generales bilingües en inglés y español, consideramos pertinente, en aras de dotar a nuestro trabajo de una mayor exhaustividad, acotar el campo de aplicación a dos diccionarios generales bilingües que cumplen una serie de criterios científicos, de manera que podamos llevar a cabo de un modo más eficaz el contraste de los resultados entre las obras seleccionadas.

En consecuencia, hemos optado por limitar nuestro estudio a las últimas ediciones publicadas de dos diccionarios considerados entre los más prestigiosos en el campo de la lexicografía bilingüe de inglés y de español, puesto que proceden de editoriales con una larga trayectoria en la aplicación de los avances de la lexicografía a sus obras:

-Collins Universal Español-Inglés, English-Spanish, de ahora en adelante CU. 
-Gran Diccionario Oxford: Español-Inglés, Inglés-Español, de ahora en adelante GDO.

Tanto el $C U$ como el GDO cuentan con un largo recorrido que avala su pertinencia para constituir el campo de aplicación de nuestro estudio. De hecho, hay que remontarse a 1971 para constatar la publicación de la primera edición del CU, como señala Hastings (2000: 206) y, desde entonces, se han sucedido nueve ediciones. A pesar de que la primera edición del GDO es posterior, puesto que fue lanzada en 1994, tenemos constancia de que han visto la luz cuatro ediciones. Con estas cifras queda demostrado el impacto editorial y probada la extensa difusión de los mencionados diccionarios en el contexto hispanohablante, entre otras razones, por la implantación incontestable de la lengua inglesa como segunda lengua en todos estos países.

De todos modos, la delimitación de nuestro campo de aplicación fue una decisión que no tomamos a la ligera, puesto que dicha selección se asienta en los resultados de trabajos previos, como el dirigido por Corpas et al. (2001: 248), en el que se corrobora que las obras que forman parte de nuestro corpus de diccionarios son las más utilizadas por los estudiantes de traducción: "en cuanto a los bilingües con el español, los alumnos de inglés (B) suelen tener el Oxford, el Collins o ambos". A esta conclusión llegaron tras analizar las respuestas obtenidas a partir de una serie de cuestionarios que completaron los alumnos de la licenciatura de Traducción de la Universidad de Málaga.

Otro trabajo de investigación que llega a la misma conclusión fue el realizado por Fernández Quesada (2009: 149-162), quien al investigar los errores de traducción de una muestra formada por estudiantes de lenguas vuelve a incidir en que los diccionarios más utilizados por éstos son "el Gran Diccionario Oxford (2005) y el Collins Universal (2008)".

Por otra parte, esta realidad no nos sorprende, dado que desde un primer acercamiento a ambas obras constatamos que reúnen una serie de requisitos marcados por los expertos (Roberts, 1997; Landau, 2001: 7-42) como elementos que asisten a los usuarios a la hora de elegir de un determinado diccionario; es decir, estas obras se ajustan a una serie de parámetros que, a nuestro juicio, explicarían su éxito editorial y permitirían realizar un análisis contrastivo de la información en ellos recogida.

Dichos parámetros podrían resumirse, en primer lugar, en su orientación hacia un amplio espectro de usuarios potenciales, en presentar un tamaño que podría definirse como "college dictionary", en ofrecer cobertura de las principales variedades diatópicas del inglés y del español, y en presentarse en soporte electrónico, el GDO en CD-ROM y el $C U$ en un archivo que se descarga de internet a partir de la clave inserta en la solapa del volumen en papel. Por último, en el prestigio de la editorial que, en 
principio, avala la fiabilidad de la información en ellos contenida, puesto que para su elaboración los lexicógrafos se han basado en ediciones precedentes que han revisado y complementado con unidades léxicas extraídas de corpus lingüísticos.

Por tanto, una vez precisado el campo de aplicación en el que realizar nuestro estudio, procedemos a describir la metodología empleada para analizar cómo se representa y caracteriza el saber especializado en los mencionados diccionarios.

3. LA GESTIÓN DEL CONOCIMIENTO ESPECIALIZADO EN LOS DICCIONARIOS GENERALES BILINGÜES: METODOLOGÍA DE ANÁLISIS

A pesar de que por el presumible carácter "general" recogido en la denominación de los diccionarios generales bilingües se podría pensar que en estas obras solamente se ofrece cobertura del léxico general, la realidad es que el empleo de "general" incluye "una selección representativa de unidades léxicas pertenecientes a distintos niveles lingüísticos (literario, estándar, coloquial) y subconjuntos de la lengua" (Haensch, 1997: 148).

En esta línea, los diccionarios generales bilingües incluyen USE, que suelen distinguirse del léxico general mediante el marcado diatécnico, como así lo corroboran numerosos autores (Thoiron, 1998; Boulanger, 2001; Roberts, 2004; Roberts y Josselin, 2005; Josselin, 2005). No obstante, dicho marcado puede realizarse mediante el empleo de una amplia variedad de recursos:

Además de las abreviaturas, pues, los principales recursos lexicográficos que se utilizan para indicar el campo temático de uso y procedencia de una voz especializada son:

a) los segmentos discursivos del tipo "se aplica en...", "en...";

b) una palabra clave entre paréntesis;

c) el campo explicitado entre paréntesis;

d) la utilización de hipónimos;

e) el nombre científico (Estopà, 1998: 381).

A la vista de la infinidad de recursos existentes, para proceder al análisis de la caracterización del saber especializado en nuestra selección de diccionarios, en primer lugar, revisaremos el apartado de la "Ayuda" de dichas obras para comprobar si se ofrecen datos respecto al mecanismo utilizado para marcar diatécnicamente las USE y distinguirlas del léxico general.

Asimismo, constataremos qué forma adopta dicho recurso en cada uno de los diccionarios generales bilingües seleccionados, por si pudieran coexistir varios recursos de marcado dentro de una misma obra, dado que algunos autores (Jessen, 1996: 181-182; Josselin, 2005: 324) concluyen 
que en los diccionarios generales bilingües no siempre se observa la deseada coherencia y transparencia en lo que respecta al marcado del léxico especializado.

Una vez conocido el recurso de marcado empleado y la forma que adopta procederemos a extraer las diferentes áreas del saber a las que pertenecen las USE recogidas en la nomenclatura de los diccionarios generales bilingües de nuestro corpus.

Además, consultaremos en el apartado de la "Ayuda" si se precisan los límites relativos a dichas áreas y si se explican las relaciones de inclusión entre los diversos campos del saber; dimensiones ambas que han sido objeto de estudio en varios de los trabajos referidos anteriormente para otros pares de lenguas (Thoiron, 1998: 647; Boulanger, 2001: 260).

Ante la convicción generalizada manifestada y compartida por todos los autores mencionados de que los detalles ofrecidos en los diccionarios son a todas luces insuficientes para determinar y perfilar los campos del saber en ellos recogidos, tras extraer las áreas de conocimiento representadas, los límites entre las mismas así como su distribución conceptual, proponemos llevar a cabo un análisis comparado de los mencionados campos del saber con la estructuración de los mismos propuesta en el Tesauro de la UNESCO. El recurrir a esta obra de referencia nos parece pertinente porque se caracteriza por ser la autoridad mundial en este campo y presumiblemente su etiquetado y su marcado puede ser tomado como marco de referencia por cualquier editorial de prestigio. Estamos convencidos de que esta propuesta nos permitirá una aproximación rigurosa a la hora de estudiar cómo se vertebra el saber especializado recogido en los diccionarios generales bilingües, y facilitará la obtención de datos fiables referentes a la gestión de dicho saber en los mencionados diccionarios.

\section{LA CARACTERIZACIÓN DEL SABER ESPECIALIZADO EN EL CU}

Primeramente procedemos a revisar el apartado de la "Ayuda", constatando que no se ofrece ninguna indicación del procedimiento empleado por los lexicógrafos para diferenciar las USE del léxico general. EI único atisbo por el que inferimos que en la nomenclatura se ofrece cobertura de las USE nos remite a la sección titulada "Abreviaturas", donde se exponen una serie de etiquetas y, tras un escrutinio de las mismas, podemos afirmar que muchas de ellas hacen referencia a campos de especialidad de los que presumiblemente se ofrece cobertura: 


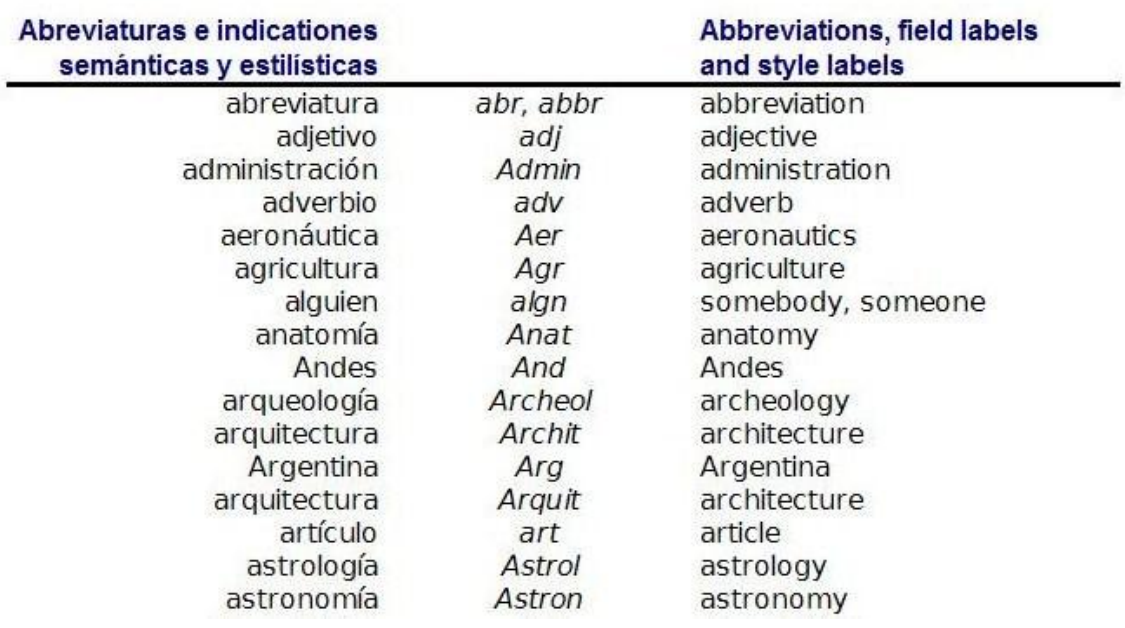

Fragmento del listado de abreviaturas presentado en el CU

No obstante, consideramos coherente cotejar si las abreviaturas propuestas se emplean como marcas de especialidad en el artículo lexicográfico en cada una de las secciones. Tomamos aleatoriamente diez abreviaturas: "Admin", "Ecol”, "Fin", "Geol", "Ling", "Min", "Pol”, "Rad", “Telec" y "Zool", las introducimos en la casilla de búsqueda de la interfaz y seleccionamos la búsqueda "en definición", primeramente en la sección de inglés-español y, a continuación, en la combinación de español-inglés.

Los resultados obtenidos son idénticos para las diez etiquetas consultadas en las dos secciones de las que se compone el $C U$ y ponen de manifiesto que el empleo de las abreviaturas tiene por función en esta obra señalar la pertenencia de un significado a un determinado campo del saber.

Una vez constatado el empleo de las MABR para marcar diatécnicamente las USE, del listado de abreviaturas expuesto en el apartado de la "Ayuda" del CU extraemos aquellas que hacen referencia a los distintos campos del saber. En total, se presentan 99 abreviaturas, aunque en algunos casos detectamos que dos abreviaturas se utilizan para hacer referencia a un mismo campo: 


\begin{tabular}{|c|c|c|c|}
\hline № & denominación en español & abreviaturas & denominación en inglés \\
\hline 1. & arquitectura & Archit, Arquit & architecture \\
\hline 2. & biología & Bio, Biol & biology \\
\hline 3. & comercio & Com, Comm & commerce, business \\
\hline 4. & costura & Cost, Sew & sewing \\
\hline 5. & escolar & Escol, Scol & school \\
\hline 6. & farmacia & Farm, Pharm & pharmacy \\
\hline 7. & ferrocarriles & Ferro, Rail & railways \\
\hline 8. & filosofía & Fil, Philos & philosophy \\
\hline 9. & física & Fís, Phys & physics \\
\hline 10. & fisiología & Fisiol, Physiol & physiology \\
\hline 11. & fotografía & Fot, Phot & photography \\
\hline 12. & informática & Inform, Comput & computing \\
\hline 13. & matemáticas & Mat, Math & mathematics \\
\hline 14. & mecánica & Mec, Mech & mechanics \\
\hline 15. & meteorología & Met, Meteo & meteorology \\
\hline 16. & mitología & Mit, Myth & mythology \\
\hline 17. & música & Mús, Mus & music \\
\hline 18. & náutica & Náut, Naut & nautical \\
\hline 19. & óptica & Ópt, Opt & optics \\
\hline 20. & psicología & Psic, Psych & psychology \\
\hline 21. & química & Quím, Chem & chemistry \\
\hline 22. & teatro & Teat, Theat & theatre \\
\hline 23. & Téc, Tech & technical \\
\hline 24. & Typography \\
\hline
\end{tabular}

Campos del saber con dos abreviaturas en el CU 
En consecuencia, el número de campos del saber a los que pertenecen las USE incluidas en la nomenclatura del $C U$ queda reducido a 75. Una vez conocido el número de áreas del saber de las que se ofrece cobertura en el $\mathrm{CU}$, procedemos a analizar la descripción de los límites entre dichas áreas, así como los datos relativos a las relaciones de inclusión de las mismas.

Tal y cómo esperábamos, tras examinar minuciosamente todos los detalles ofrecidos en el apartado de la "Ayuda" constatamos que no se ofrece ningún dato en el que se ponga de manifiesto cómo han procedido los lexicógrafos a fijar las denominaciones de los campos del saber cubiertos por esta obra.

Asimismo, tampoco hemos hallado informaciones respecto a los criterios empleados para establecer las fronteras entre los distintos campos del saber, ni tampoco ninguna descripción en la que se evidencie cómo se ha procedido a establecer las relaciones de inclusión entre dichos campos.

Conscientes de que el análisis probablemente pudiera no revelar datos iluminadores de la organización del conocimiento especializado, procedemos a realizar un análisis comparado de los campos del saber recogidos en el $\mathrm{CU}$ con la estructuración de los mismos propuesta en el Tesauro (UNESCO, 2008).

Para llevar a cabo esta comparativa insertamos una a una las denominaciones de las áreas de conocimiento de las que presumiblemente se ofrece cobertura en el $\mathrm{CU}$ en el buscador del Tesauro y confeccionamos un esquema siguiendo las relaciones de inclusión ofrecidas por esta obra de referencia, de modo que hemos coloreado en rojo aquellos campos que funcionan como hiperónimos ${ }^{1}$ en el Tesauro y que, sin embargo, se omiten ${ }^{2}$ en el $C U$.

\footnotetext{
1 En el Tesauro de la UNESCO están recogidos como "TG", sigla que significa término genérico.

2 El tema "7 Países y agrupaciones de países" no lo hemos incluido porque no existe ningún campo asociado al mismo.
} 
1. EDUCACTÓN

1.1. ESTABI.ECTMIENTOS DE ENSENANZA 1.1.1. UNIVERSIDAD

1.2. PROFESIONALES DE LA EDUCACIÓN

121 FSCOL AR

1.3. MATERIAS DE ENSENANZA BÁSICA 1.3.1.COCTNA

2. CIENCIA

2.1. MATEMÁTICAS 2.1.1.GEOMETRIA

2.2. Quimica

2.3. CIENCIAS DEL ESPACIO

2.3.1.ASTRONOMIA

2.4. GEOGRAFIA

2.5. METEOROLOGIA

2.6. CIENCLAS AMBIENTAL.ES F

INGENIERIA

2.6.1. ECOLOGIA

2.7. CIENCIAS DE LA TIERRA

2.7.1.GEOLOGIA

2.8. BIOLOGIA

2.8.1.ANATOMEA

28.2 FISIOLOGIA

2.9. CIENCIAS NATURAL.ES

2.9.1.BOTÁNICA

29.27 OOLOGIA

2.10. MEDICTNA

IEDICINA

2.10.1. FARMACIA

2.10.2. VETERINARIA

2.11 . FISICA

2.11.1. OPTICA
2.11 .2 MECANICA 2.11.21. EI.ECTRICIDAD

3. CULTURA

$$
\begin{aligned}
& \text { 3.1. FILOSOFIA } \\
& \text { 3.2. HISTORIA } \\
& \text { 3.2.1.MTIOLOGIA } \\
& \text { 3.2.2.ARQUEOLOGIA } \\
& \text { 3.3. LINGOISTICA } \\
& \text { 3.3.1.GRAMATICA } \\
& \text { 3.3.2.FONETTICA } \\
& \text { 3.4. LITERATURA } \\
& \text { 3.5. REL.IGION } \\
& \text { 3.5.1.ASTROLOGIA } \\
& \text { 3.6. ARTES ESCENCAS } \\
& \text { 3.6.1.MUSICA } \\
& \text { 3.6.2.TEATRO } \\
& \text { 3.6.3.CINE } \\
& \text { 3.7. ARTES VISUALES } \\
& \text { 3.7.1.ARQUTTECTURA } \\
& \text { 3.7.2.FOTOGRAIIA }
\end{aligned}
$$

3.8. OCto

3.8.1.DFPORTE

3.8.1.1. FÜrBOI

4. CIFNCIAS SOCIAIES Y HUMANAS

4.1. PSICOLOGIA

4.2 CIENCIAS SOCIAIFS

4.21.SOCIOLOGLA

5. INFORMACION Y COMUNICACTON

5.1. INDUSTRIA DE LA INFORMACIÓN

5.1.1.RADIO

S.1.2 TELEVISTON

5.1.3.METODO DE IMPRESIÓN

5.1.3.1. TLPOGRAFIA

5.2. CIENCLAS DE L.A INFORMACION 5.2.1.INFORMÁTICA

5.3. TECNOLOGIADEIA

TNFORMACION

5.3.1.TELECOMUNCACIÓN

6. POLITICA, DERECHO Y ECONOMIA (3)

6.1. AGRICULTURA

6.1.1.HORTICULTURA

6.2. POLITICA Y GOBIERNO

6.21.PARI AMENTO

6.3. INDUSTRLA

6.4. FINANZAS Y COMFRCIO(2) 6.4.1.BOLSA

6.5. ORGANIZACTÓN Y GESTIÓN 6.5.1.ADMINISTRACTÓN

6.6. EOUIPOS E INSTAL ACIONES 6.6.1.AUTOMOVTLISMO

6.7. INGENTERIA DE LA INDUSTRLA Y DE LOS TRANSPORTES

6.7.1.METALURGIA

6.8. NVGENTERIA DE MINAS, CTVII. Y MII.TAR

6.8.1.MINERIA

6.8.2AGRIMENSURA

Organización conceptual de los campos del saber del CU siguiendo el modelo propuesto en el Tesauro (UNESCO, 2008) 
No obstante, conviene señalar que varios de los campos del saber de los que se ofrece cobertura en el $C U$ no se contemplan en el Tesauro (UNESCO, 2008); tales como "aeronáutica", "construcción", "costura", "esquí", "ferrocarriles", "militar", "náutica", "tauromaquia" y "técnico".

Además, si agrupamos las distintas áreas de conocimiento especializado en los siete temas principales en los que el Tesauro organiza el saber experto, podemos observar que las áreas relativas a la "ciencia" (30\%) y a la "cultura" (23\%) agrupan un mayor número de campos, si bien ello no implica que sean los campos con un mayor número de entradas marcadas diatécnicamente.

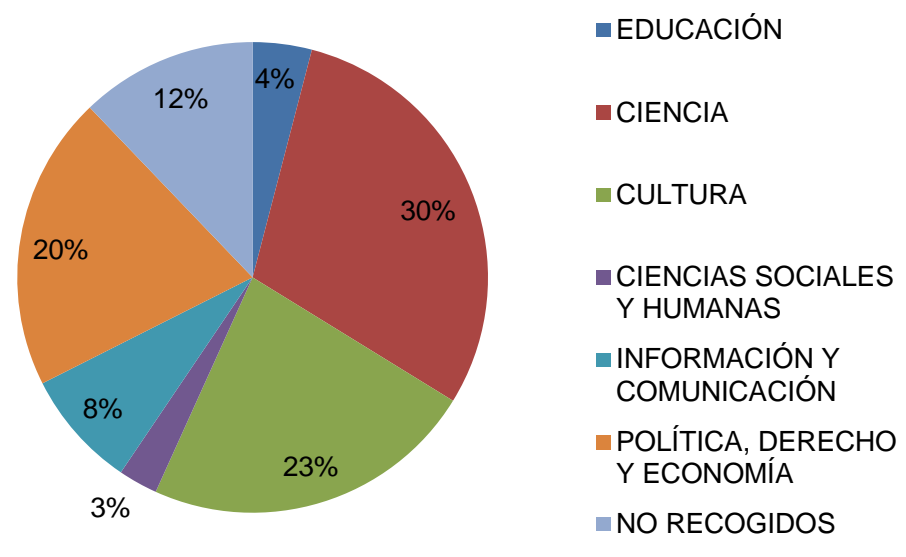

Distribución de los campos según los temas recogidos por la UNESCO en el CU

\section{LA CARACTERIZACIÓN DEL SABER ESPECIALIZADO EN EL GDO}

Siguiendo un procedimiento de análisis similar al efectuado en el $C U$, en primer lugar procedemos a revisar el apartado de la "Ayuda" del GDO para comprobar si se ofrecen datos relativos al recurso empleado para marcar diatécnicamente las USE.

En dicho apartado, en la sección titulada "Estructura del artículo lexicográfico en español-inglés" se explica que una de las partes de las que se compone el artículo lexicográfico son los "indicadores", siendo una de las categorías la que hace referencia a las áreas de conocimiento, denominada "indicadores de campo semántico":

3 Esta área de conocimiento podría estar recogida como concepto hipónimo de "OCIO>DEPORTE". 


\author{
field labels \\ cápsula $f$ \\ indicadores de campo semántico \\ A (Farm) capsule \\ B. (Bot) capsule \\ C. (Audio) cartridge \\ D) (Espac) capsule
}

Descripción de los indicadores de campo semántico en el GDO (2008: xi)

Asimismo, esta información vuelve a reproducirse en la sección dedicada a la "Estructura del artículo lexicográfico en Español-Inglés":

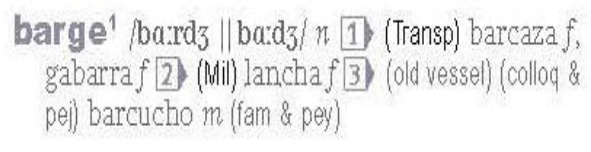

\section{indicadores de campo semántico}

field labels

Descripción de los indicadores de campo semántico en el GDO (2008: xvi

A la vista de estos hechos convendrá cotejar la coherencia de estas propuestas en la "Ayuda" con su utilización en el diccionario. Para ello, del listado ofrecido en la sección de "Abreviaturas" escogemos aleatoriamente diez: "Adm", "Ecol", "Fin", "Geol", "Ling", "Min", "Pol", "Rad", "Telec" y "Zool", las introducimos en el motor de búsqueda de la interfaz, activamos la correspondencia exacta y realizamos la búsqueda primeramente en la sección de inglés-español y, a continuación, en la combinación lingüística de español-inglés. Los resultados obtenidos son idénticos en las dos secciones, poniéndose de manifiesto que el motor de búsqueda no ha encontrado coincidencias.

Teniendo en cuenta estos resultados consideramos pertinente repetir la búsqueda con semejantes parámetros, pero ahora introduciendo las denominaciones completas en lengua española correspondientes a las abreviaturas anteriores en la sección de español-inglés: "Administración", "Ecología", "Finanzas", "Geología", "Lingüística", "Minería", "Política", "Radio", "Telecomunicaciones" y "Zoología".

En este caso, los resultados obtenidos son idénticos y ponen en evidencia que el recurso empleado en esta obra para marcar el léxico especializado se corresponde con la denominación completa del campo del saber. Para confirmar estos resultados, repetimos la búsqueda en la combinación de inglés-español introduciendo los equivalentes en inglés de las denominaciones de los mencionados campos del saber.

Los resultados ofrecidos por la interfaz son similares a los obtenidos en la sección homóloga y vuelven a poner de manifiesto el empleo de las 
denominaciones completas del campo como recurso para marcar diatécnicamente las USE.

En consecuencia, del listado de abreviaturas con sus denominaciones ofrecido en el apartado de la "Ayuda" procedemos a extraer las denominaciones en las lenguas inglesa y española que hacen referencia a los campos del saber de los que presumiblemente se ofrece cobertura.

\section{Abbreviations/Abreviaturas}

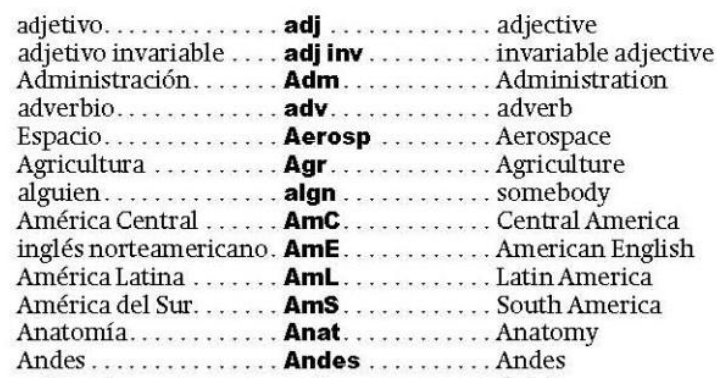

Fragmento del listado de abreviaturas presentado en el GDO

En dicho listado observamos que la marca "Textiles" se emplea en ambas secciones, de manera que el número de marcas asciende a 187, ofreciéndose en esta obra cobertura de un total de 94 campos del saber.

Una vez conocidos los campos del saber a los que pertenecen las USE recogidas en la nomenclatura, examinamos las informaciones recogidas en el apartado de la "Ayuda" del GDO y, tal y como ocurre en el $C U$, podemos afirmar que en esta obra no se ofrece ningún dato que describa cómo han procedido los compiladores a fijar las denominaciones de los campos del saber representados en dicho diccionario, ni tampoco hemos hallado informaciones que aporten criterios de cómo se ha llevado a cabo el establecimiento de los límites entre los distintos campos del saber de los que se ofrece cobertura.

En consecuencia, siguiendo el mismo procedimiento de análisis que el realizado con el $C U$, comparamos la organización de las áreas de conocimiento del GDO con la estructuración propuesta en el Tesauro. 

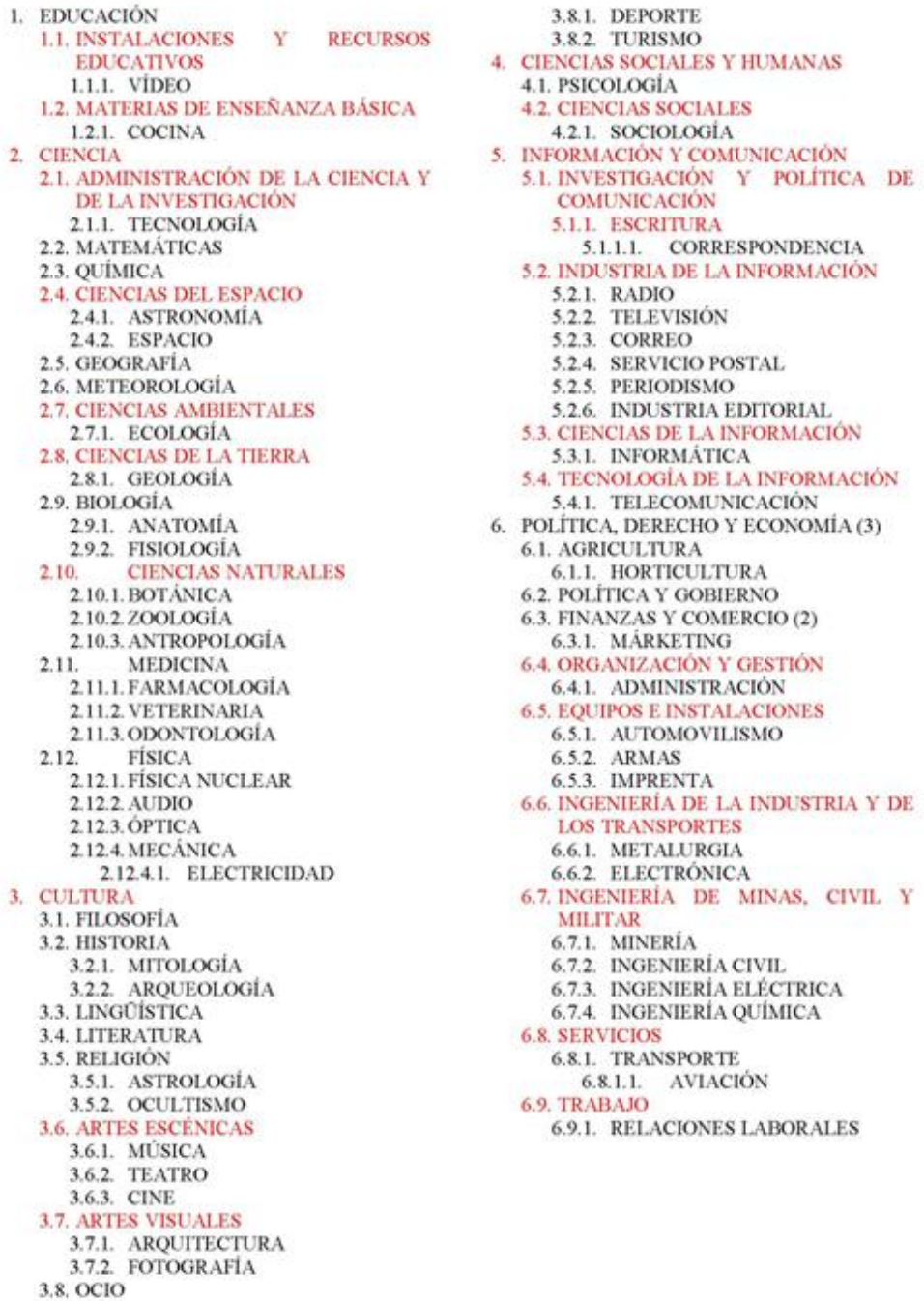

Organización conceptual de los campos del saber del GDO siguiendo el modelo propuesto por el Tesauro

Tal y como sucede en el $C U$, varios de los campos marcados en el GDO no se recogen en el Tesauro (UNESCO, 2008); por ejemplo, "biblia", "construcción", "equitación", "espectáculos", "ferrocarriles", "fisco", "indumentaria", "ingeniería", "juegos tradicionales", "militar", "náutica", 
"servicios sociales", "tauromaquia", "textiles" y "vinicultura". Además, observamos una cierta falta de sistematicidad en la denominación y extensión conceptual de alguno de los campos, dado que coexisten campos como "indumentaria" y "textiles", que podrían considerarse afines entre sí.

Siguiendo con la terminología de la UNESCO categorizamos las áreas de especialidad de las que se ofrece cobertura según los siete temas principales en los que esta institución organiza el conocimiento especializado.
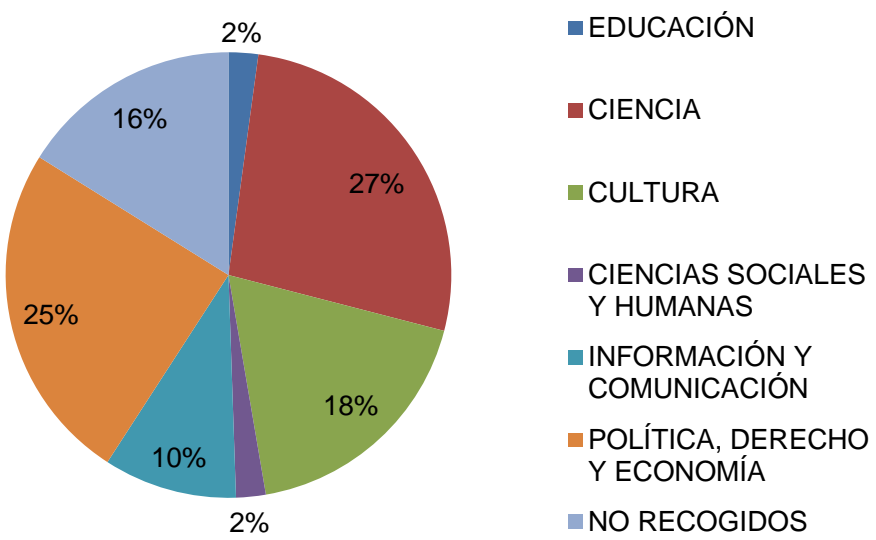

Distribución de los campos según los temas recogidos por la UNESCO en el GDO

En el gráfico se pone de manifiesto que los temas con un mayor número de campos del saber en esta obra son "ciencia" (27\%) y "política, derecho y economía" (25\%).

1. Comparativa entre el $C U$ y el $G D O$

Una vez realizado el análisis de las áreas de conocimiento representadas en cada uno de los diccionarios generales bilingües de nuestro corpus y de los criterios relativos al establecimiento de los límites entre los distintos campos del saber de los que se ofrece cobertura, podemos analizar comparativamente los resultados obtenidos en las obras que forman parte de nuestro campo de aplicación.

Respecto al recurso de marcado empleado para etiquetar las USE, los lexicógrafos que elaboran el $C U$ han optado por utilizar las MABR en tanto que sus homólogos del GDO han preferido el uso de las MCAM.

Por otro lado, desde un punto de vista cuantitativo observamos diferencias respecto al número de campos de los que se ofrece cobertura 
en los diccionarios seleccionados, dado que en el GDO se ofrece cobertura a 19 campos más que en el $C U$, obra en la que solamente se recogen 75 áreas de especialidad.

Por lo que concierne a la denominación de las áreas de conocimiento, 58 de los campos cubiertos tienen denominaciones semejantes, tal y como puede consultarse en el Apéndice I.

Conviene apuntar que en dicha relación de campos del saber únicamente hemos incluido aquellas áreas de conocimiento cuyas denominaciones coinciden con exactitud y omitimos otras, tales como "industria" (CU) e "industria editorial" $(G D O)$ o "farmacia" $(C U)$ y "farmacología" $(G D O)$, dado que entendemos que dichas denominaciones no hacen referencia al mismo campo del saber, sino a disciplinas próximas al mismo.

Por otro lado, la cobertura dada a ciertos campos del saber es diferente en ambas obras, como se puede constatar en las siguientes tablas:

\begin{tabular}{|l|l|l|l|}
\hline № & denominación en español & abreviatura & denominación en inglés \\
\hline 1. & aeronáutica & Aer & aeronautics \\
\hline 2. & costura & Cos/Sew & sewing \\
\hline 3. & escolar & Escol/Scol & school \\
\hline 4. & farmacia & Farm & pharmacy \\
\hline 5. & fútbol & Ftbl & football \\
\hline 6. & geometría & Geom & geometry \\
\hline 7. & gramática & Gram & grammar \\
\hline 8. & industria & Ind & industry \\
\hline 9. & parlamento & Parl & parliament \\
\hline 10. & fonética & Phon & phonetics \\
\hline 11. & ciencia & Sci & science \\
\hline 12. & esquí & Ski & skiing \\
\hline 13. & bolsa & St Ex & stock exchange \\
\hline 14. & agrimensura & Survey & surveying \\
\hline 15. & técnico & Téc, Tech & technical \\
\hline
\end{tabular}




\begin{tabular}{|l|l|l|l|} 
16. & tipografía & Tip/Typ & typography \\
\hline 17. & universidad & Univ & university \\
\hline
\end{tabular}

Áreas de conocimiento cubiertas únicamente en el CU

\begin{tabular}{|c|c|c|}
\hline № & Denominación en español & Denominación en inglés \\
\hline 1. & antropología & anthropology \\
\hline 2. & armas & arms \\
\hline 3. & arte & art \\
\hline 4. & audio & audio \\
\hline 5. & aviación & aviation \\
\hline 6. & biblia & bible \\
\hline 7. & correo & post \\
\hline 8. & correspondencia & correspondence \\
\hline 9. & electrónica & electronics \\
\hline 10. & equitación & equestrianism \\
\hline 11. & espacio & aerospace \\
\hline 12. & espectáculos & entertainment \\
\hline 13. & farmacología & pharmacology \\
\hline 14. & fisco & $\operatorname{tax}$ \\
\hline 15. & física nuclear & nuclear physics \\
\hline 16. & imprenta & printing \\
\hline 17. & imprenta e industria editorial & printing and publishing \\
\hline 18. & indumentaria & clothing \\
\hline 19. & industria editorial & publishing \\
\hline 20. & ingeniería & engineering \\
\hline 21. & ingeniería civil & civil engineering \\
\hline 22. & ingeniería eléctrica & electrical engineering \\
\hline
\end{tabular}

Hikma 13 (2014), 97-123 


\begin{tabular}{|l|l|l|}
\hline 23. & ingeniería química & chemical engineering \\
\hline 24. & juegos & games \\
\hline 25. & márketing & marketing \\
\hline 26. & ocio & leisure \\
\hline 27. & ocultismo & occult \\
\hline 28. & odontología & dentistry \\
\hline 29. & relaciones laborales & labor relations \\
\hline 30. & servicios sociales & social administration \\
\hline 31. & tecnología & technology \\
\hline 32. & textiles & textiles \\
\hline 33. & transporte & transport \\
\hline 34. & turismo & tourism \\
\hline 35. & vídeo & video \\
\hline 36. & vinicultura & wine \\
\hline
\end{tabular}

Áreas de conocimiento cubiertas únicamente en el GDO

Analizado cada diccionario en sí mismo, tal y como se puede observar en los siguientes gráficos, frente a la realidad de un $77 \%$ de campos del saber comunes frente a un $23 \%$ de campos solo recogidos en el $C U$, en el GDO tenemos un $62 \%$ de campos comunes y un $28 \%$ de campos únicamente cubiertos por esta obra: 


\section{$C U$}

a campos del saber comunes a campos del saber diferentes

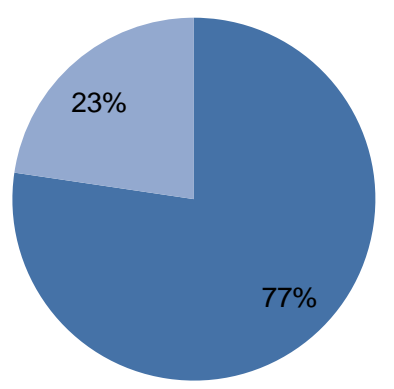

Áreas de conocimiento comunes y diferenciadas cubiertas en el CU

\section{GDO}

n campos del saber comunes campos del saber diferentes

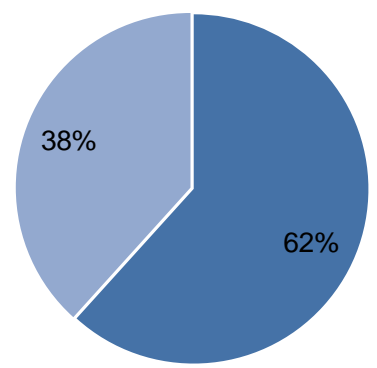

Áreas de conocimiento comunes y diferenciadas cubiertas el GDO

Por tanto, la representatividad de los campos recogidos en ambas obras sobre el total de áreas de las que se ofrece cobertura en cada una de las obras es mayor en el $C U$ que en el GDO.

Por lo que respecta a la comparación de las diversas áreas de conocimiento cubiertas en cada uno de los diccionarios generales bilingües de nuestro corpus siguiendo la estructuración del Tesauro (UNESCO, 2008) 
y una vez expuestas las distintas relaciones de inclusión entre campos, conviene señalar lo siguiente: un aspecto que se reproduce tanto en el $\mathrm{CU}$ como en el GDO es el relacionado con la omisión de aquellos campos del saber que funcionan como conceptos hiperónimos de otros subcampos de los que sí que ofrece cobertura; por ejemplo, el campo de las "ciencias naturales", que se corresponde con uno de los microtesauros del Tesauro, se omite tanto en el $C U$ como en el GDO. En cambio, en ambas obras se incluye el subcampo de la "zoología", que es una de las ramas en las que se vertebra las "ciencias naturales", e incluso en el CU se recoge el área de la "ornitología" dentro del campo de la "zoología".

Sospechamos que la causa de que se produzca este fenómeno pudiera residir en que las denominaciones de los conceptos superordinados probablemente sean de interés general, de modo que los lexicógrafos que compilan el $C U$ y el GDO consideran que no es necesario recogerlo marcado diatécnicamente y, en consecuencia, las unidades léxicas que denominan conceptos relativos a estos campos están incluidos en la nomenclatura de los diccionarios generales bilingües de nuestra selección sin marca de especialidad, de manera que dicho campo del saber estará representado en la nomenclatura sin marcar diatécnicamente.

\section{CONCLUSIONES \\ La gestión del conocimiento especializado en los diccionarios} generales bilingües seleccionados se caracteriza por la escasa sistematicidad, puesto que las escasas informaciones recogidas en el apartado de la "Ayuda" muestran incoherencias con el procedimiento de marcado empleado en el artículo lexicográfico. En este sentido, en el GDO se expone que se utilizan las abreviaturas (MABR) en los preliminares y en realidad se opta por el uso de las denominaciones (MCAM). Esta contradicción podría estar motivada por el hecho de que los diccionarios en formato electrónico seleccionados no son elementos aislados, sino que se venden conjuntamente con la versión en papel y su función podría ser servir de material de apoyo a los usuarios, de modo que su empleo no es independiente. No obstante, conviene mencionar que Rodríguez Reina (2002: 252) ya precisó en su estudio que en los diccionarios generales bilingües se desatienden aspectos como la descripción de la selección y del empleo de las marcas de materia. Además, coincidimos con Roberts (2004: 138 ) en poner de relieve la escasa estandarización en cuanto al recurso utilizado para marcar diatécnicamente el léxico especializado, puesto que en una de las obras (el $\mathrm{CU}$ ) se emplean las abreviaturas (MABR) en tanto que en la segunda de las obras (el GDO) se utilizan las denominaciones (MCAM) de las áreas de especialidad cubiertas. En consecuencia, los 
usuarios y, en especial, los traductores en formación, tendrán que estar avezados en el manejo de la herramienta que consulten.

Por otro lado, no se siguen planteamientos rigurosos a la hora de asignar una denominación a un área de especialidad, ni tampoco existe un posicionamiento de los compiladores de los diccionarios generales bilingües analizados al respecto. En este sentido, en los diccionarios generales bilingües de nuestra selección ofrecen algunas denominaciones de los campos del saber que no se recogen en la obra de referencia por excelencia, el Tesauro (UNESCO, 2008), poniéndose de manifiesto la falta de sistematicidad a la hora de denominar los diversos campos de saber, de manera que los traductores encontrarán diferente información según la obra que consulten, por lo que recomendamos complementar la información obtenida con el empleo de otras herramientas.

Asimismo, ciertas áreas genéricas se omiten en el $C U$ y en el $G D O$, probablemente porque el léxico perteneciente a dichas áreas es muy conocido por los usuarios, de manera que consideran que no es necesario marcarlo diatécnicamente. En consecuencia, una de las causas por las que el léxico especializado no recibe un tratamiento sistemático en los diccionarios generales bilingües de inglés-español y de español-inglés podría estar constituida por la anarquía existente a la hora de denominar las diversas áreas de conocimiento a las que pertenece el léxico especializado recogido en la nomenclatura de dichas obras de referencia.

En lo relativo a la selección de las áreas de conocimiento cubiertas en los diccionarios generales bilingües destacamos que no se lleva a cabo con la deseada coherencia, dado que solamente dos tercios de los campos del saber coinciden en los diccionarios generales bilingües de nuestro corpus. Por tanto, en función de la obra que los usuarios consulten encontrarán entradas marcadas que, aun perteneciendo al mismo campo de especialidad, estarán categorizadas en campos diferentes. Este hecho puede causar confusión, especialmente a los traductores en formación, que no dominan los campos del saber en los que se estructura el conocimiento especializado. Nuestra recomendación va encaminada a corroborar los datos obtenidos empleando otros recursos como las bases de datos multilingües, los diccionarios monolingües o los corpus lingüísticos.

Para finalizar, nos gustaría destacar que la cobertura de los diversos campos del saber en los que se estructura el conocimiento experto debería normalizarse, de manera que quede constancia en el apartado de la "Ayuda" de los diccionarios generales bilingües los criterios que se han seguido para establecer los límites entre los distintos campos del saber de los que se ofrece cobertura en los diccionarios generales bilingües de inglés y de español. También deberían ofrecerse indicaciones de cómo se fijan las relaciones de inclusión entre los mencionados campos del saber. Una vez 
más, estas propuestas no son novedosas, sino que siguen la línea iniciada en los trabajos de Jessen (1996: 180) y Boulanger (2001:252) también mostraron su disconformidad al respecto. No obstante, conviene apuntar que de las deficiencias descritas se derivan implicaciones directas para los usuarios que consultan estas obras. Por sus características, consideramos que los diccionarios generales bilingües seleccionados deberían de cubrir campos idénticos con denominaciones semejantes y presentando equilibrio desde el punto de vista cuantitativo, de manera que existiera cierta estandarización en torno a los campos cubiertos.

REFERENCIAS BIBLIOGRÁFICAS

ATKINS, B. T. Sue; VARANTOLA, Krista, "Language Learners Using Dictionaries: The Final Report on the EURALEX/AILA Research Project on Dictionary Use". En: Using Dictionaries: Studies of Dictionary Use by Language Learners and Traslators. Tübingen: Max Niemeyer Verlag, 1998, pp. 21-81.

_-, "Monitoring Dictionary Use". En: Using Dictionaries: Studies of Dictionary Use by Language Learners and Translators. Tübingen: Max Niemeyer Verlag, 1998, pp. 83-122.

BOULANGER, Jean-Claude, "L'aménagement des marques d'usage technolectales dans les dictionnaires généraux bilingues". En: Les dictionnaires de la langue française. Paris: Honoré Champion, 2001, pp. 247-271.

BOWKER, Lynne, "Meeting the Needs of Translators in the Age of eLexicography". En: Electronic Lexicography. Oxford: Oxford University Press, 2012, pp. 379-397.

CORPAS PASTOR, Gloria; LeIVA RoJo, Jorge; VARELA SALINAS, María-José, "El papel del diccionario en la formación de traductores e intérpretes: análisis de necesidades y encuestas de uso". En: Diccionarios y enseñanza. Alcalá de Henares: Universidad de Alcalá, 2001, pp. 239273.

DURÁN MUÑOZ, Isabel, "Specialised Lexicographical Resources: a Survey of Translators' Needs". En eLexicography in the 21st century: New Challenges, New applications. Proceedings of ELEX 2009, Cahiers du Cendal. Louvain-la-Neuve: Presses Universtaires de Louvaine-LaNeuve, 2010, pp. 55-66.

EstopÀ, Rosa, "El léxico especializado en los diccionarios de lengua general: las marcas temáticas". En: Revista española de lingüística, 28, 2, 1998, pp. 359-388.

FERNÁNDEZ QUESADA, Nuria, "El diccionario bilingüe y los errores de traducción en los estudiantes de inglés como lengua 'c'". En: Investigación lexicográfica para la enseñanza de lenguas. Málaga: 
Servicio de Publicaciones de la Universidad de Málaga, 2009, pp. 149-163.

GallimberTI, Beatriz; Russel, Roy (eds.), Gran Diccionario Oxford: EspañolInglés, Inglés-Español. 4 ${ }^{\mathrm{a}}$ ed. Oxford: Oxford University Press, 2008.

HAENSCH, Günther, Los diccionarios del español en el umbral del siglo XXI: Problemas actuales de la lexicografía. Salamanca: Universidad de Salamanca, 1997.

HASTINGS, Andrew, "El diccionario inglés-español, español-inglés como herramienta para el traductor". En: Documentación, terminología y traducción. Madrid: Síntesis, 2000, pp. 109-122.

JeSSEN, Anette, The Presence and Treatment of Terms in General Dictionaries. Tesis Doctoral. Ottawa: University of Ottawa, 1996. Disponible en línea en: http://www.ruor.uottawa.ca/en/handle/10393/4427 [Fecha de consulta: 22 de septiembre de 2014].

Josselin, Amélie, Place et rôle des terminologies dans les dictionnaires généraux unilingues et bilingues. Étude d'un domaine de spécialité: Volcanologie. Tesis Doctoral. Lyon: Université Lumière Lyon II, Centre de Recherche en Terminologie et Traduction, 2005. Disponible en línea en:

http://theses.univ-

lyon2.fr/documents/lyon2/2005/josselin a\#p=0\&a=top

[Fecha de consulta: 22 de septiembre de 2014].

LANDAU, Sydney I., Dictionaries. The Art and Craft of Lexicography. 2nd Edition. Cambridge: Cambridge University Press, 2001

MACKINTOSH, Kristen, "An Empirical Study of Dictionary Use in L2-L1 Translation". En: Using Dictionaries: Studies of Dictionary Use by Language Learners and Translators. Tübingen: Max Niemeyer Verlag, 1998, pp. 123-149.

MEYER, Ingrid, "The General Bilingual Dictionary as a Working Tool in 'Thème'". En: Meta, 33, 3, 1988, pp. 368-376.

Ortego Antón, María Teresa, Estudio contrastivo inglés/español del tratamiento del léxico especializado recogido en los diccionarios generales bilingües: Collins Universal y Gran Diccionario Oxford. Tesis Doctoral. Soria: Universidad de Valladolid, 2012. Disponible en línea en http://uvadoc.uva.es/handle/10324/2022 [Fecha de consulta: 22 de septiembre de 2014].

ROBERTS, Roda P., "Using Dictionaries Efficiently". En: 38th Annual Conference of the American Translators Association. San Francisco, California, 1997.

"Terms in General Dictionaries". En: A New Spectrum of Translation Studies. Valladolid: Universidad de Valladolid, 2004, pp. 121-140. 
Roberts, Roda P.; Josselin, Amélie, "Le traitement des termes dans les dictionnaires généraux". En: De la mesure dans les termes. Hommage à Philippe Thoiron. Lyon: Presses Universitaires de Lyon, 2005, pp. 324-348.

RODRíGUEZ REINA, Ma Pilar, "Las marcas de especialidad: Una cuenta pendiente de difícil solución". En: Panorama actual de la terminología, Granada: Comares, 2002, pp. 327-357.

Schriven, Rob; Love, Catherine; MiOt-Cadley, Gaëlle (eds.), Collins Universal Español-Inglés, English-Spanish, 9ª ed. Barcelona: Random House Mondadori/Glasgow: Harper Collins Publishers, 2009.

SIERRA SORIANO, Ascensión, El diccionario bilingüe. Estructura y nomenclatura. Alicante: Editorial Club Universitario, 2001.

THOIRON, Philippe, "Place et rôle de la terminologie dans les dictionnaires bilingues non spécialisés. Le cas de la terminologie médicale dans le Dictionnaire Hachette-Oxford (français-anglais)". En: Mots chiffrés et déchiffrés. Paris: Honoré Champion, 1998, pp. 621-650.

UNESCO, 2008. Tesauro. Disponible en línea en:

http://databases.unesco.org/thessp/ [Fecha de consulta: 22 de septiembre de 2014]. 


\section{APÉNDICE}

\begin{tabular}{|c|c|c|c|c|c|}
\hline \multirow[b]{2}{*}{ № } & \multicolumn{3}{|c|}{$C U$} & \multicolumn{2}{|c|}{ GDO } \\
\hline & $\begin{array}{l}\text { denominación } \\
\text { del campo en } \\
\text { español }\end{array}$ & abr. & $\begin{array}{l}\text { denominación } \\
\text { del campo en } \\
\text { inglés }\end{array}$ & $\begin{array}{l}\text { denominación del } \\
\text { campo en español }\end{array}$ & $\begin{array}{l}\text { denominación } \\
\text { del campo en } \\
\text { inglés }\end{array}$ \\
\hline 1. & administración & Admin & administration & administración & administration \\
\hline 2. & agricultura & Agr & agriculture & agricultura & agriculture \\
\hline 3. & anatomía & Anat & anatomy & anatomía & anatomy \\
\hline 4. & arqueología & $\begin{array}{c}\text { Arche } \\
\text { ol }\end{array}$ & archeology & arqueología & archeology \\
\hline 5. & arquitectura & $\begin{array}{l}\text { Archit, } \\
\text { Arquit }\end{array}$ & architecture & arquitectura & architecture \\
\hline 6. & astrología & Astrol & astrology & astrología & astrology \\
\hline 7. & astronomía & Astron & astronomy & astronomía & astronomy \\
\hline 8. & $\begin{array}{l}\text { automóviles, } \\
\text { automovilismo }\end{array}$ & Aut & automobiles & automovilismo & cars \\
\hline 9. & biología & $\begin{array}{l}\text { Bio, } \\
\text { Biol }\end{array}$ & biology & biología & biology \\
\hline 10. & botánica & Bot & botany & botánica & botany \\
\hline 11. & cine & Cine & cinema & cine & cinema \\
\hline 12. & culinario, cocina & Culin & culinary, cooking & cocina & cookery \\
\hline 13. & comercio & $\begin{array}{l}\text { Com, } \\
\text { Com } \\
m\end{array}$ & $\begin{array}{l}\text { commerce, } \\
\text { business }\end{array}$ & comercio & business \\
\hline 14. & construcción & $\underset{r}{\text { Const }}$ & construction & construcción & building \\
\hline 15. & deportes & Dep & sport & deporte & sport \\
\hline 16. & $\begin{array}{l}\text { derecho, } \\
\text { jurídico }\end{array}$ & Jur & law, legal & derecho & law \\
\hline 17. & ecología & Ecol & ecology & ecología & ecology \\
\hline 18. & economía & Econ & economy & economía & economics \\
\hline 19. & educación & Educ & education & educación & education \\
\hline 20. & electricidad & Elec & electricity & electricidad & electricity \\
\hline
\end{tabular}

Hikma 13 (2014), 97-123 


\begin{tabular}{|c|c|c|c|c|c|}
\hline 21. & ferrocarriles & $\begin{array}{l}\text { Ferr, } \\
\text { Rail }\end{array}$ & railways & ferrocarriles & railways \\
\hline 22. & filosofía & $\begin{array}{c}\text { Fil, } \\
\text { Philos }\end{array}$ & philosophy & filosofía & philosophy \\
\hline 23. & finanzas & Fin & finance & finanzas & finance \\
\hline 24. & física & $\begin{array}{l}\text { Fís, } \\
\text { Phys }\end{array}$ & physics & física & physics \\
\hline 25. & fisiología & $\begin{array}{c}\text { Fisiol, } \\
\text { Physi } \\
\text { ol }\end{array}$ & physiology & fisiología & physiology \\
\hline 26. & fotografía & $\begin{array}{l}\text { Fot, } \\
\text { Phot }\end{array}$ & photography & fotografía & photography \\
\hline 27. & geografía & Geog & geography & geografía & geography \\
\hline 28. & geología & Geol & geology & geología & geology \\
\hline 29. & gobierno & Govt & government & gobierno & government \\
\hline 30. & historia & Hist & history & historia & history \\
\hline 31. & horticultura & Hort & horticulture & horticultura & horticulture \\
\hline 32. & informática & $\begin{array}{c}\text { Comp } \\
\text { ut, } \\
\text { Inform }\end{array}$ & computing & informática & computing \\
\hline 33. & lingüística & Ling & linguistics & lingüística & linguistics \\
\hline 34. & literatura & Literat & literature & literatura & literature \\
\hline 35. & matemáticas & $\begin{array}{l}\text { Mat, } \\
\text { Math }\end{array}$ & mathematics & matemáticas & mathematics \\
\hline 36. & mecánica & $\begin{array}{l}\text { Mec, } \\
\text { Mech }\end{array}$ & mechanics & mecánica & $\begin{array}{l}\text { mechanical } \\
\text { engineering }\end{array}$ \\
\hline 37. & medicina & Med & medicine & medicina & medicine \\
\hline 38. & metalurgia & Metal & metallurgy & metalurgia & metallurgy \\
\hline 39. & meteorología & $\begin{array}{l}\text { Met, } \\
\text { Meteo }\end{array}$ & meteorology & meteorología & meteorology \\
\hline 40. & militar & Mil & military & militar & military \\
\hline 41. & minería & Min & mining & minería & mining \\
\hline
\end{tabular}




\begin{tabular}{|c|c|c|c|c|c|}
\hline 42. & mitología & $\begin{array}{l}\text { Mit, } \\
\text { Myth }\end{array}$ & mythology & mitología & mythology \\
\hline 43. & música & $\begin{array}{l}\text { Mús, } \\
\text { Mus }\end{array}$ & music & música & music \\
\hline 44. & náutica & $\begin{array}{l}\text { Náut, } \\
\text { Naut }\end{array}$ & nautical & náutica & nautical \\
\hline 45. & óptica & $\begin{array}{l}\text { Opt, } \\
\text { Opt }\end{array}$ & optics & óptica & optics \\
\hline 46. & ornitología & Orn & ornithology & periodismo & journalism \\
\hline 47. & política & Pol & politics & política & politics \\
\hline 48. & psicología & $\begin{array}{l}\text { Psic, } \\
\text { Psych }\end{array}$ & psychology & psicología & psychology \\
\hline 49. & química & $\begin{array}{l}\text { Chem } \\
\text { Quím }\end{array}$ & chemistry & química & chemistry \\
\hline 50. & radio & Rad & radio & radio & radio \\
\hline 51. & religión & Rel & religion & religión & religion \\
\hline 52. & sociología & Sociol & sociology & sociología & sociology \\
\hline 53. & tauromaquia & Taur & bullfighting & tauromaquia & bullfighting \\
\hline 54. & teatro & $\begin{array}{l}\text { Teat, } \\
\text { Theat }\end{array}$ & theatre & teatro & theater \\
\hline 55. & $\begin{array}{c}\text { telecomunicacio } \\
\text { nes }\end{array}$ & Telec & $\begin{array}{c}\text { telecommunication } \\
s\end{array}$ & telecomunicaciones & $\begin{array}{c}\text { telecommunication } \\
s\end{array}$ \\
\hline 56. & televisión & $T V$ & television & televisión & television \\
\hline 57. & veterinaria & Vet & $\begin{array}{l}\text { veterinary } \\
\text { medicine }\end{array}$ & veterinaria & veterinary science \\
\hline 58. & zoología & Zool & zoology & zoología & zoology \\
\hline
\end{tabular}

Áreas de conocimiento comunes en el CU y en el GDO 